\title{
Integrated simulation of ELM triggered by pellet through energy absorption and transport enhancement
}

\author{
N. Hayashi 1), V. Parail 2), F. Koechl 3), N. Aiba 1), T. Takizuka 1), S. Wiesen 4), \\ P.T. Lang 5), N. Oyama 1), T. Ozeki 1) and JET EFDA Contributors*
}

1) Japan Atomic Energy Agency, Naka, Ibaraki-ken 311-0193, Japan

2) EURATOM/CCFE Fusion Association, Culham Science Centre, Abingdon OX14 3DB, UK

3) Association EURATOM-ÖAW/ATI, Atominstitut, TU Wien, 1020 Vienna Austria

4) EURATOM Association Forschungszentrum Juelich GmbH, Institute for Energy Research, IEF-4, Plasmaphysics, D-52425 Juelich, Germany

5) Max-Planck-Institut fur PlasmaPhysik, EURATOM Association, Boltzmannstrasse 2, 85748 Garching, Germany

E-mail: hayashi.nobuhiko@jaea.go.jp

\begin{abstract}
Two integrated core / scrape-off-layer (SOL) / divertor transport codes TOPICS-IB and JINTRAC with links to MHD stability codes have been coupled with models of pellet injection to clarify effects of pellet on the behavior of edge localized modes (ELMs). The energy absorption by pellet and its further displacement due to ExB drift as well as transport enhancement by the pellet were found to be able to trigger the ELM. The ablated cloud of pellet absorbs the background plasma energy and causes the radial redistribution of pressure due to the subsequent ExB drift. On the other hand, the sharp increase in local density and temperature gradients in the vicinity of ablated cloud could cause transient enhancement of heat and particle transport. Both mechanisms produce a region of an increased pressure gradient in the background plasma profile within the pedestal, which triggers the ELM. The mechanisms have the potential to explain a wide range of experimental observations.
\end{abstract}

\section{Introduction}

The energy loss caused by edge localized modes (ELMs) is crucial for the erosion of divertor plates, the plasma confinement and the control for the steady state in tokamaks. The pellet injection is considered as one possible method to increase the ELM frequency and to reduce energy loss during the ELM. A few ideas have been already explored in attempts to explain ELM triggering by pellet $[1,2]$. The triggering mechanisms, however, are not fully understood yet.

Multi-machine experiments showed a wide range of phenomena, which follow pellet ablation [1,3-7]. This includes very prompt ELM onset at the time when the pellet passes the pedestal region [5], ability to trigger the ELM with the high repetition rate by the pellet injected from any location [1], about $10 \%$ decrease in the electron temperature along the pellet penetration path without the density increase [6], and magnetic perturbation induced by the pellet observed even in non-ELMing H-mode and L-mode plasmas [3,7]. The last two phenomena are proposed as candidates of ELM triggering mechanism, which triggers fast global perturbation spreading over the whole flux surface with the electron thermal or Alfven speed [7]. Experimental observations also indicate that pellet ablation clouds may absorb the energy of background plasma and transport it along the major radius via vertical drift. On the other hand, the sharp increase in local density and temperature gradients close to an ablated cloud generate a wide spectrum of MHD perturbations, which could temporarily increase local heat and particle transports in the background plasma. The aim of this paper is to model predictively these mechanisms and find out if they can reproduce the main experimental observations.

\footnotetext{
${ }^{*}$ See the Appendix of F. Romanelli et al., paper OV/1-3, this conference
} 
The integrated simulation code is one of the most effective methods to study the ELM mechanism $[2,8,9]$. For example, an integrated code COCONUT, which couples a 1.5 dimensional (1.5D) core transport code JETTO with a 2D scrape off layer (SOL) / divertor code EDGE2D and is now included into larger suite of transport and MHD codes JINTRAC (JET INtegrated suite of TRAnsport Codes) [10], has been fully tested and is extensively used for the study of ELM dynamics, such as the mechanism of transition from conductive to convective energy loss during ELM [8]. On the other hand, an integrated code TOPICS-IB [9] was coupled with a dynamic five-point model for SOL / divertor plasmas and a stability code for peeling-ballooning modes, MARG2D. The TOPICS-IB is based on a 1.5D core transport code TOPICS extended to the integrated simulation for burning plasmas. The TOPICS-IB was recently used to clarify the physical mechanism of the ELM energy loss, such as the collisionality dependence of the energy loss caused by the edge bootstrap current and the SOL transport [9].

In this paper, two integrated core / SOL / divertor transport codes TOPICS-IB and JINTRAC with links to MHD stability codes have been coupled with models of pellet injection to clarify effects of pellet on the ELM behavior. By using both integrated codes, we study two mechanisms of ELM triggering by the pellet, i.e., pellet energy absorption and transport enhancement effects. TOPICS-IB and JINTRAC complement each other in many respects. Simultaneous use of both codes to simulate the same mechanism provides the extra benefit of code benchmarking and ensures consistency of obtained results.

\section{Integrated modeling of pellet triggered ELM with TOPICS-IB and JINTRAC}

The mechanism of pellet triggered ELM is investigated by integrated codes TOPICS-IB and JINTRAC. Details of both codes are shown in $[8,9,10]$. Both codes are coupled with respective pellet models. Some essential features are explained as follows.

\subsection{TOPICS-IB}

TOPICS-IB is based on a 1.5D core transport code TOPICS, which is coupled with a linear MHD stability code MARG2D, a SOL / divertor five-point model and a pellet model APLEX (Ablated PeLlet with ExB drift). TOPICS solves the 1D transport and current diffusion equations on the normalized toroidal flux coordinate, $\rho$, and the Grad-Shafranov equation of MHD equilibrium on the $2 \mathrm{D}$ plane $(\mathrm{R}, \mathrm{Z})$. Particle and thermal diffusivities consist of neoclassical and anomalous ones where the anomalous diffusivities are given as empirical formulas. In order to produce the H-mode pedestal, the transport near the edge is reduced to the neoclassical value calculated by the matrix inversion method with a prescribed pedestal width, $\Delta_{\text {ped }}$. The ELM model is developed by coupling TOPICS with MARG2D. In the present simulation, the stability of $n=1-50$ modes are examined at given time-intervals, where $n$ is the toroidal mode number. When unstable modes appear, an ELM is assumed to occur. The ELM enhanced diffusivities are added on the basis of eigenfunction profiles of unstable modes, where the maximum value, $\chi_{\mathrm{ELM}}{ }^{\max }$, is given as a parameter. The ELM enhanced transport is maintained for a time interval $\tau_{\mathrm{ELM}}$ given as a parameter. The five-point model is based on time-dependent Braginskii's fluid equations. Particle flux and heat fluxes across the separatrix obtained in TOPICS are used as inputs and the five-point model calculates the SOL density and temperatures at the separatrix which are used as boundary conditions in TOPICS. The parameters are chosen as $\Delta_{\mathrm{ped}}=0.05$ on $\rho, \chi_{\mathrm{ELM}}{ }^{\max }=100 \mathrm{~m}^{2} / \mathrm{s}$ and $\tau_{\mathrm{ELM}}=200 \mu \mathrm{s}$ in this paper. 
APLEX model is based on equations of pellet ablation, ExB drift of detached plasma cloud (or plasmoid) and cloud energy absorption. The reduction rate of pellet radius $r_{p}$ by the ablation is given by the neutral gas and plasmoid shielding (NGPS) description as [11],

$$
\frac{d r_{p}}{d t}=-C_{p} \frac{n_{e \infty}^{1 / 3} T_{e \infty}^{11 / 6}}{4 \pi n_{\text {solid }} Z^{2 / 3} W^{1 / 3} r_{p}^{2 / 3}\left(\ln \Lambda_{e n}\right)^{2 / 3}}
$$

The meaning of each variable is the same as in the reference and omitted here due to the page limitation. The time derivative of detached cloud speed $v_{\perp}$ in the $\mathrm{R}$ direction is given as [11-13],

$$
\Sigma_{\rho} \frac{d v_{\perp}}{d t}=\frac{2}{R}\left(p_{c}-p_{\infty}\right) Z_{c} \alpha\left(Z_{c}\right)-\max \left(\frac{2 B^{2} v_{\perp}}{\mu_{0} c_{A \infty}}, H\left(t-\tau_{\sigma}\right) \frac{\sigma B^{2}\left(1-e^{-t / \tau_{c}}\right) L_{b} L_{I \mathrm{P}} v_{\perp}}{2\left(L_{\phi}-Z_{c}\right)}\right)
$$

The first term in right-hand-side of eq. (2) is a driving term due to the ExB drift and second and third terms are damping terms. When the cloud plasma pressure equilibrates with the background one, $\mathrm{p}_{\mathrm{c}}=\mathrm{p}_{\infty}$, the cloud is homogenized with the background plasma. To study the transport enhancement effect during the homogenization, the ad-hoc diffusivity is added to the background plasma transport based on a Gaussian profile. The time derivative of cloud energy $\mathrm{E}_{\mathrm{c}}$ is given as $[13,14]$,

$$
\frac{d E_{c}}{d t}=f_{s} f_{d} \int q_{e \mathrm{P}} d S_{\mathrm{P}}+\int q_{e \perp} d S_{\perp}-\left(p_{c}-p_{\infty}\right) c_{s} S_{\perp}
$$

where the sheath shielding factor $f_{s}=0.2$ and $f_{d}$ denotes the deposition factor [15]. It is worth noting that background plasma transports use 1D models even during the pellet ablation and further drift, and the background plasma stability is examined in 1D approximation as well.

\subsection{JINTRAC}

JINTRAC includes the pellet ablation and deposition code HPI2, the 1.5D core transport code JETTO and the multi-fluid SOL-divertor code EDGE2D-EIRENE [8,10]. In JETTO, the transport equations are solved for plasma current, temperatures and density. Transport coefficients in the core are calculated according to the mixed Bohm/gyroBohm transport model. The pedestal is established by transport reduction to the neoclassical level within the edge barrier with a prescribed barrier width. If the normalized pressure gradient $\alpha$ exceeds critical value $\alpha_{\text {crit }}$ (prescribed by a value obtained from the MHD stability code or an experimentally-evaluated value) anywhere within the pedestal, ELM events are emulated by a temporary sharp increase in edge transport. In the SOL, perpendicular transport is defined by the diffusivities at the separatrix; longitudinal transport follows Braginskii's approximation.

HPI2 determines the pellet particle source by application of a pellet ablation model which is based on the NGPS description. The $\mathrm{E} \times \mathrm{B}$ drift of the cloudlets can be taken into account following a four-fluids Lagrangian model for the plasmoid homogenisation process [13]. The structure of the drift equation is similar to (2):

$$
\frac{d v_{\perp}}{d t}=\frac{1}{1+\left(1-P_{A l f}-P_{c o n}\right)\left(L_{c o n}^{\prime} n_{\infty} / Z_{c} n_{c}\right)}\left\{\frac{2\left(p_{c}-p_{\infty}\right)}{n_{c} m_{p} R} \alpha\left(Z_{c}\right)-\frac{v_{\perp} B^{2}}{n_{c} m_{p} Z_{c}} \cdot\left[P_{A l f} \frac{2}{\mu_{0} c_{A \infty}}+P_{c o n} \frac{\left[1-e^{-t /\left(\tau_{c}+\tau_{s e l f}\right)}\right] \pi R_{0}^{2} \sigma}{L_{c o n}}\right]\right\}
$$

A detailed description of (4) is given in [16]. In HPI2, the heat transfer from the background plasma to the plasmoid is governed by the following equations, which describe the change in electron and ion energy content of the plasmoid [13]: 


$$
\frac{d E_{c}^{e / i}}{d t}=Q_{\infty c}^{e / i}+Q_{c}^{i e / e i}+\frac{1}{N_{c}} \frac{d N_{c}}{d t} E_{c}^{e / i}+W_{c}^{e / i}-p_{c}^{e / i} S_{\perp} \frac{d Z_{c}}{d t}
$$

The first term describes the heat exchange between the plasmoid and the background plasma in the magnetic flux surface shell, where the plasmoid is located. The other terms in (5) account for electron-ion energy equilibration, and losses due to convection, viscosity and expansion work.

HPI2 is more detailed in comparison with APLEX model in TOPICS-IB by the following points; (1) treating cloud ion temperature $\mathrm{T}_{\mathrm{ci}}$ and electron one $\mathrm{T}_{\mathrm{ce}}$ separately (also taking account of ion heat flux) $\left(\mathrm{T}_{\mathrm{ci}}=\mathrm{T}_{\mathrm{ce}}\right.$ assumed and no ion heat flux in APLEX), (2) taking account of detailed perpendicular heat flux (constant fraction of $\mathrm{q}_{\mathrm{e} / /}$ assumed in APLEX), (3) taking account of expansion of cloud radius (constant cloud radius assumed in APLEX).

\section{Integrated simulation results}

TOPICS-IB and JINTRAC complement each other in many respects. Simultaneous use of both codes to simulate the same mechanism provides the extra benefit of code benchmarking and ensures consistency of obtained results. Parameters used in simulations are as follows; $R$ $=3.3 \mathrm{~m}, a=0.72 \mathrm{~m}, \kappa=1.7, \delta=0.086, I_{\mathrm{p}}=1.5 \mathrm{MA}, B_{\mathrm{t}}=3.5 \mathrm{~T}$ for the JT-60U ELMy H-mode experiment [17] and $R=2.9 \mathrm{~m}, a=0.91 \mathrm{~m}, \kappa=1.7, \delta=0.35, I_{\mathrm{p}}=1.7 \mathrm{MA}, B_{\mathrm{t}}=2.0 \mathrm{~T}$ for the JET ELMy H-mode experiment [18].

\subsection{ELM triggered by pellet energy absorption}

Figure 1 shows electron density $\mathrm{n}_{\mathrm{e}}$ profiles just before and $3 \mathrm{~ms}$ after the injection of a deuterium pellet from the high-field-side top (HFStop) with $r_{p}=0.6 \mathrm{~mm}$ in TOPICS-IB and JINTRAC simulations for the JT-60U experiment. In figure 1, the region in which the density increases qualitatively agrees with that in the experiment. The deposition depth in the TOPICS-IB simulation, which uses ExB drift, is about 0.2 in terms of $\rho$, which is close to the depth experimentally observed by the electron-cyclotron-emission measurement. The deposition depth in the JINTRAC simulation is slightly longer $(\sim 0.3)$. When the ExB drift and the time-dependent interaction between the pellet and the background plasma are switched off,

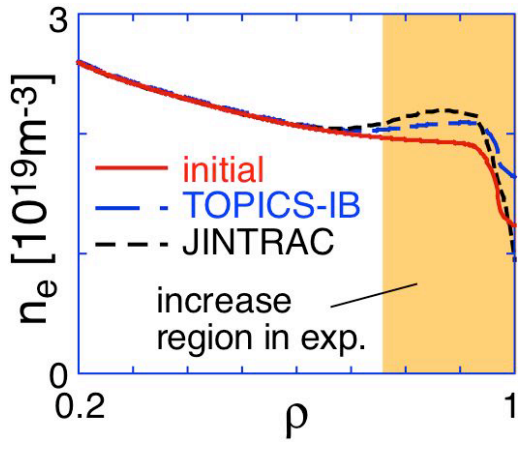

FIG. 1. $n_{e}$ profile before (red solid curve) and 3 ms after a HFStop pellet injection in TOPICS-IB and JINTRAC simulations (blue broken and black dotted curves, respectively) for JT-60U experiment where shaded area denotes densitv increase region observed in experiment. which is the same condition as the conventional ablation calculation, the deposition depth is about 0.05 in both

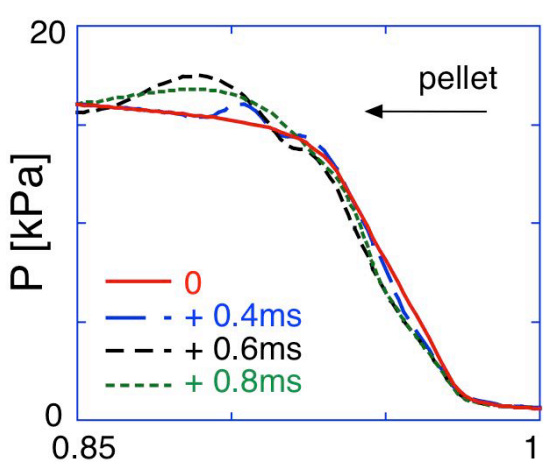

FIG. 2. Time evolution of background total pressure P profile in case of FIG. 1. 

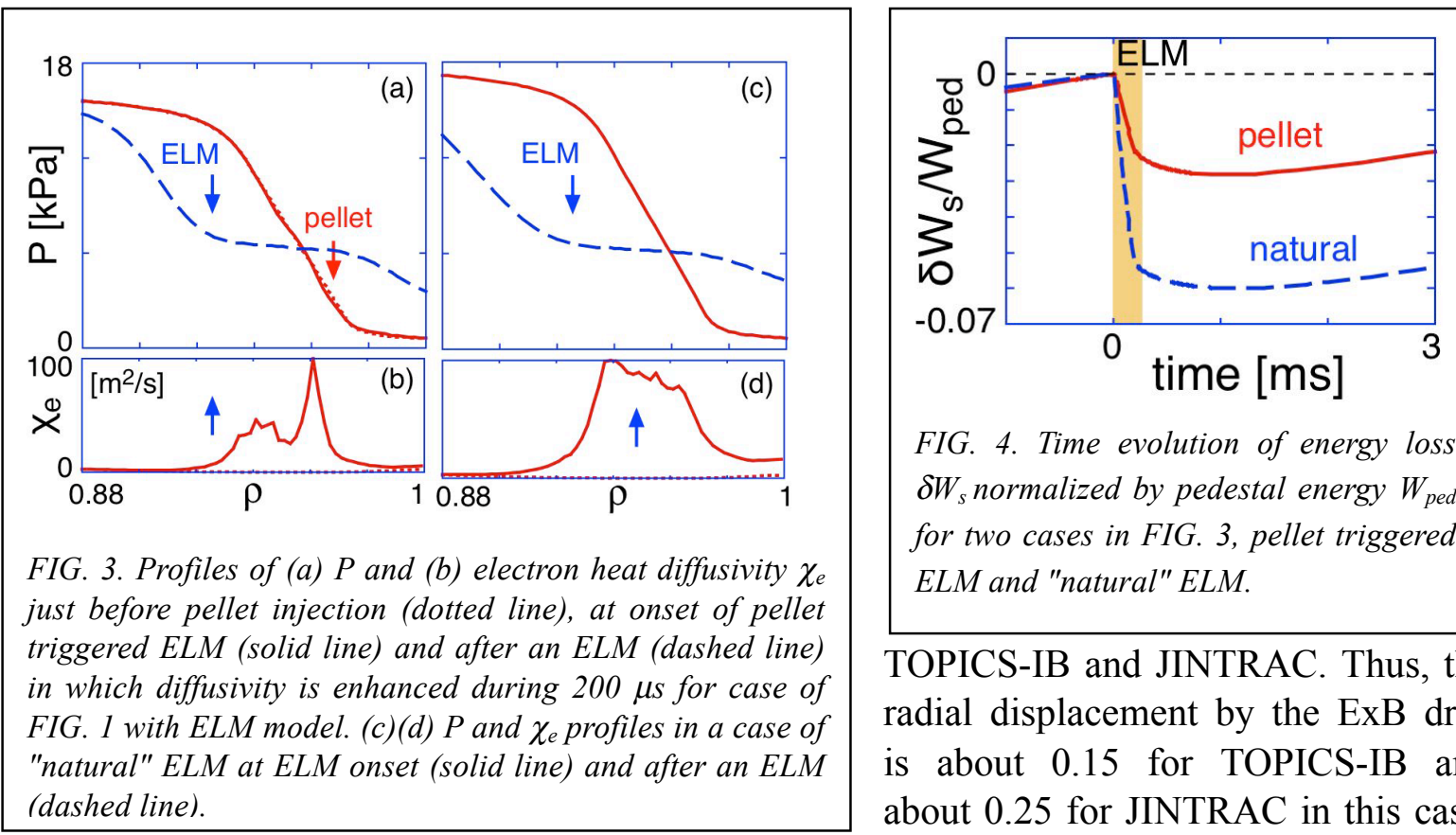

FIG. 4. Time evolution of energy loss $\delta W_{s}$ normalized by pedestal energy $W_{\text {ped }}$ for two cases in FIG. 3, pellet triggered ELM and "natural" ELM.

TOPICS-IB and JINTRAC. Thus, the radial displacement by the ExB drift is about 0.15 for TOPICS-IB and about 0.25 for JINTRAC in this case.

Figure 2 shows the time evolution of background total pressure profile in the TOPICS-IB case of figure 1, but with the ELM mode switched off. The pellet cloudlet absorbs the background plasma energy at the magnetic flux surface, which the cloudlet crosses. The ExB drift shifts inward the heated cloudlet and deposits its energy in a region, where the cloud merges with the background plasma. The energy absorption and the following ExB drift modify the background plasma profile and produce a region with steeper pressure gradient in Fig.2.

Figures 3(a) and (b) shows the same case with the ELM event enabled. The local steep pressure gradient occuring within the pedestal (indeed, local value of $\mathrm{dP} / \mathrm{d} \rho$ in Fig.3(a) exceeds that in Fig.3(c)) can destabilize the high-n ballooning mode ( $\mathrm{n}>40$ in this case) and trigger an ELM in Figs 3(a) and (b). The width of eigenfunction profile is narrower in the

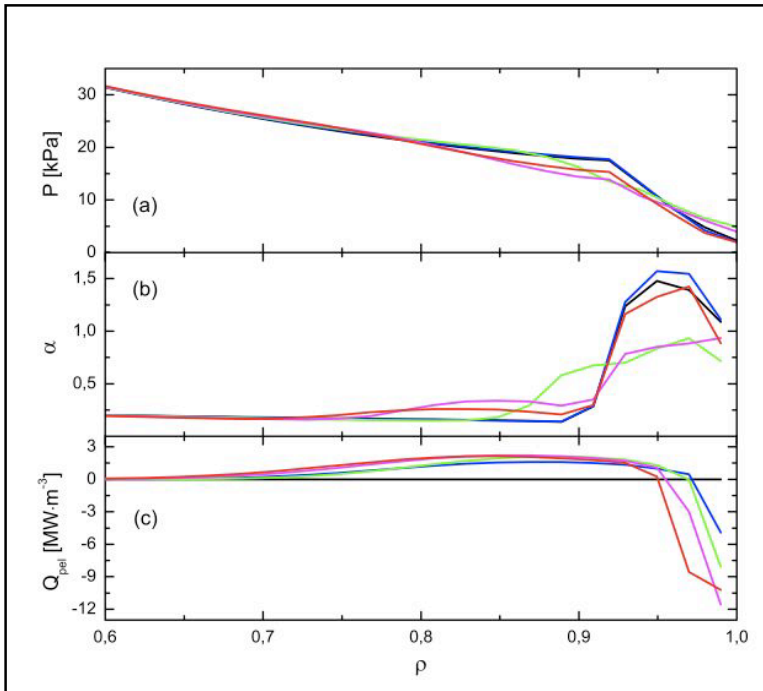

FIG. 5. Time evolution of profiles of (a) $P$, (b) $\alpha$ and (c) heat source and sink $Q_{\text {pel }}$ by VHFS pellet in a JINTRAC simulation for JET experiment without pellet transport enhancement (black: 7.3399s, blue: 7.3402s, green: 7.3405s, magenta: 7.3408s, red: $7.3411 s$. pellet triggered ELM than that in "natural" ELMs $(13 \leq n \leq 28$ unstable) in Figs $3(\mathrm{c})$ and (d). The region of ELM enhanced transport almost corresponds to the pedestal region independently of position at which the pellet

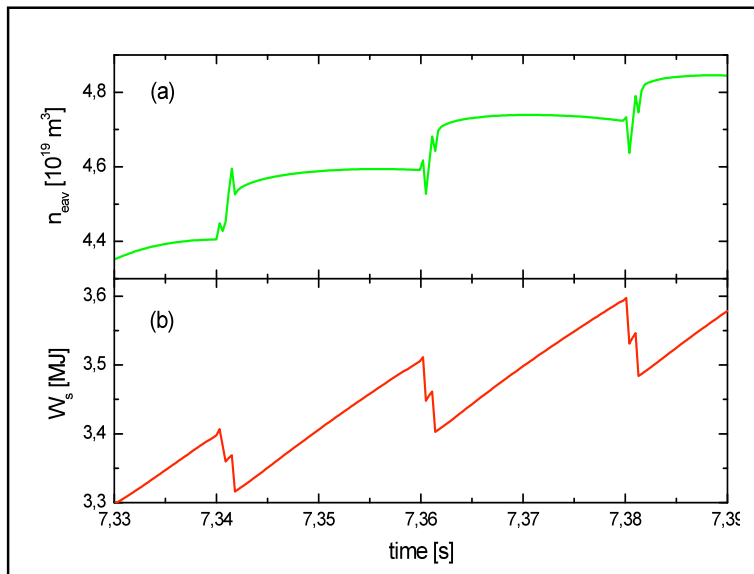

FIG. 6. Time evolution of (a) $n_{\text {eav }}$ and (b) $W_{s}$ for repetitive VHFS pellets with $50 \mathrm{~Hz}$ from $t=47.34$ s. Time evolution of profiles during first pellet is shown in FIG. 5. 
triggers the ELM. The resultant energy loss is less than half of the "natural" ELMs as shown in Fig. 4. This half reduction of ELM energy loss agrees with experimental observations $[4,5]$. We also do simulations with a low-field-side (LFS) pellet and obtain similar results.

Figure 5 shows the time evolution of profiles of (a) P, (b) $\alpha$ and (c) heat source and sink by the energy absorption effect of a vertical HFS (VHFS) pellet with $0.9 \mathrm{~mm}$ size and $150 \mathrm{~m} / \mathrm{s}$ speed in a JINTRAC simulation for the JET experiment without the pellet transport enhancement effect. The pressure redistribution by the pellet energy absorption and the subsequent ExB drift leads to an increase of pressure gradient, triggering an ELM at 7.3402 s $<\mathrm{t}<7.3404 \mathrm{~s}$. At this time, the pellet has not yet reached the pedestal top. Figure 6 shows the time evolution of (a) volume-averaged electron density $\mathrm{n}_{\text {eav }}$ and (b) stored energy $\mathrm{W}_{\mathrm{s}}$ for repetitive VHFS pellets with $50 \mathrm{~Hz}$ from $\mathrm{t}=7.34 \mathrm{~s}$. ELMs are triggered every time when pellet is injected.

\subsection{ELM triggered by pellet transport enhancement}

Figure 7 shows profiles of plasma pressure, normalized pressure gradient and ion heat diffusivity just before and $100 \mu$ s after the HFS pellet injection in a JINTRAC simulation without the energy absorption effect for the JET experiment. In the simulation, to study the pellet transport enhancement effect, the instantaneous pellet deposition is assumed and the transport is enhanced in the vicinity of deposition region for $100 \mu$ s following the deposition. In the model of pellet transport enhancement, the magnitude is much less than the ELM enhanced transport but larger than the stationary value, and the duration is based on the experimental observation in which ELMs were triggered about $50 \mu$ s after the pellet imposed the seed perturbation [5,7]. If the region with the enhanced transport spreads over the pedestal top, it can also lead to a sudden reduction of pedestal width. The transient transport enhancement and the resultant pedestal contraction are shown to be able to create a narrow region with the steep pressure gradient close to the pedestal top in Fig.7. This results in the

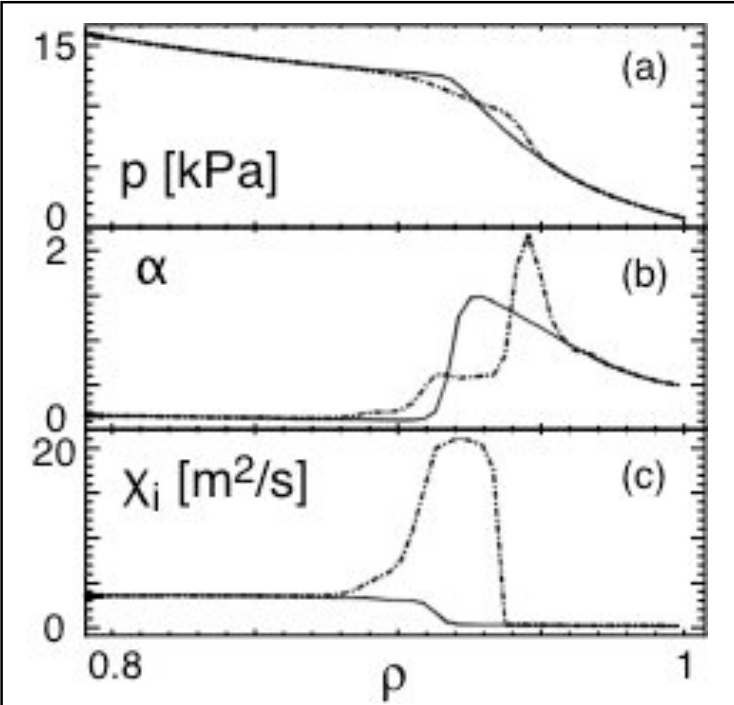

FIG. 7. Profiles of (a) P, (b) $\alpha$ and (c) ion heat diffusivity $\chi_{i}$ just before (solid line) and $100 \mu \mathrm{s}$ after (chain line) VHFS pellet injection in a JINTRAC simulation for JET experiment where transport is enhanced in vicinity of pellet deposition.

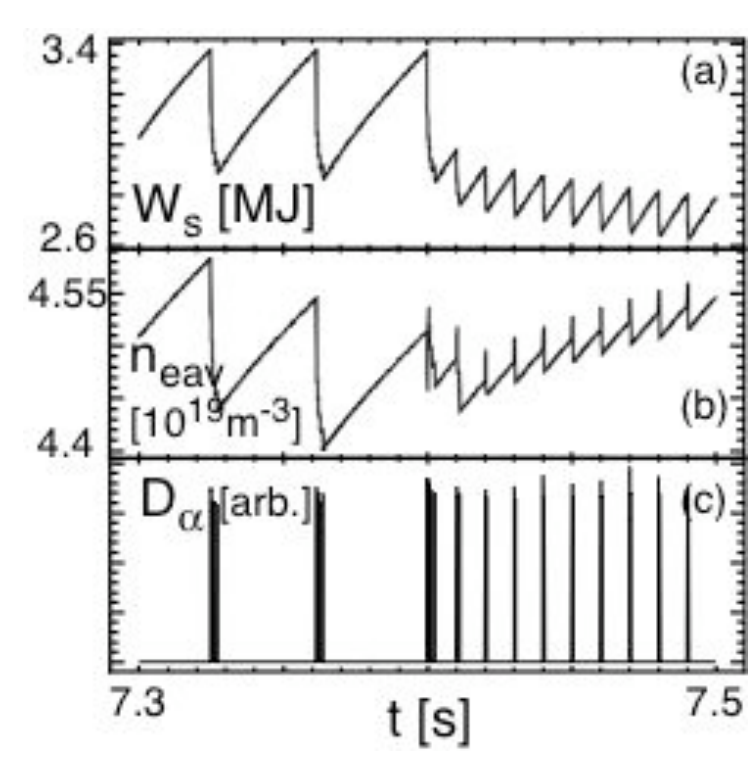

FIG. 8. Time evolution of (a) $W_{s}$, (b) $n_{\text {eav }}$ and (c) $D_{\alpha}$ emission for repetitive pellet with $100 \mathrm{~Hz}$ from $t=7.4$ $s$. ELMs before $t=7.4 \mathrm{~s}$ are "natural" ELMs. Time evolution of profiles during first pellet is shown in FIG. 7. 


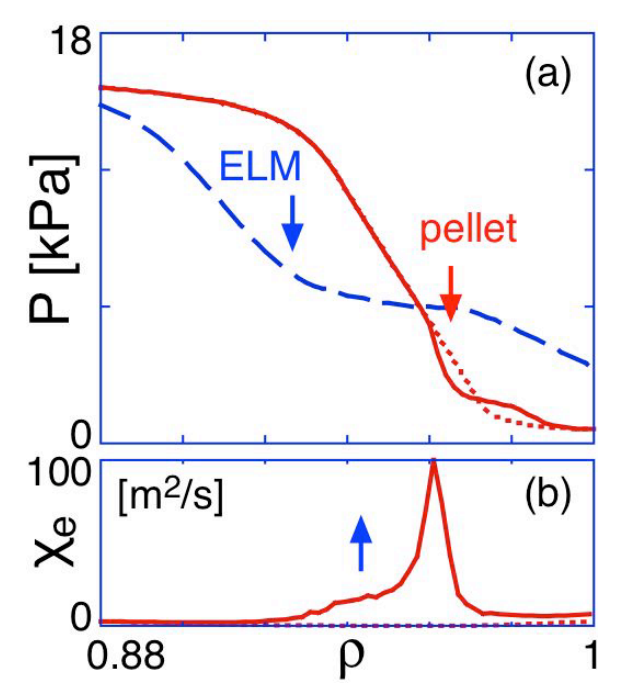

FIG. 10. Time evolution of profiles of (a) $P$ and (b) $\chi_{e}$ just before pellet injection (dotted line), at onset of pellet triggered ELM (solid line) and after an ELM (dashed line).

prompt onset of ballooning instability and ELM by using the same criteria in previous subsection. Figure 8 shows the time evolution of stored energy, volume-averaged density and from $\mathrm{t}=7.4 \mathrm{~s}$. Note that infrequent strong ELM crashes before $\mathrm{t}<7.4 \mathrm{~s}$ are due to "natural" ELMs. Each pellet triggers an ELM crash if the pellet injection frequency does not exceed $200 \mathrm{~Hz}$. ELM triggering becomes unreliable (not each pellet triggers the ELM) if pellet repetition rate exceeds $200 \mathrm{~Hz}$. Also, pellet does not reliably trigger ELM (in both experiment and modelling) if the distance between previous "natural" ELM and further pellet ablation is shorter than $\Delta \mathrm{t}<\sim 3 \mathrm{~ms}$.

Figure 9 shows the time evolution of (a) $\mathrm{P}$ and (b) $\chi_{\mathrm{e}}$ profiles with the pellet transport enhancement effect used in TOPICS-IB code for the same case as Fig. 1, but with the pellet energy absorption effect and the ELM model switched off. In this simulation it was assumed that a series of cloudlets enhances transport for $10 \mu$ s following the deposition as shown in Fig. 9(b) and creates the pressure perturbation in Fig. 9(a). Figures 10 shows the same case with the ELM event enabled. Almost the same as in Fig.3, high-n modes become unstable and those eigenfunction profiles are narrow compared with those in the "natural" ELM. The ELM energy loss triggered by the pellet is less than half of that in the "natural" ELM. Similar results are also obtained in simulations with a LFS pellet. When both the pellet energy absorption and the transport enhancement are taken into account in the TOPICS-IB simulation, the pellet makes the background pressure perturbation even stronger and triggers ELM more reliably. Either effect triggers high-n modes, with the narrowness of eigenfunction profiles and the magnitude of ELM energy loss being almost the same in both cases.

\section{Summary and discussions}


Two integrated core / SOL / divertor transport codes TOPICS-IB and JINTRAC with links to MHD stability codes have been coupled with pellet models to clarify effects of pellet on the ELM behavior. Both the energy absorption and the transport enhancement by the pellet were found to be able to trigger the ELM. The ablated cloud of pellet absorbs the background plasma energy and causes the radial redistribution of pressure due to the subsequent ExB drift. On the other hand, the sharp increase in local density and temperature gradients in the vicinity of ablated cloud causes the transient enhancement of heat and particle transport. Both mechanisms produce a region of an increased pressure gradient in the background plasma profile within the pedestal, which triggers the ELM. Simulations show that two considered mechanisms have the potential to explain a wide range of experimentally observed phenomena.

The simulation and the model validation on other sets of parameters remains as future work. Two mechanisms discussed above may fail to trigger the ELM in lower pedestal with lower pressure gradient, which appears just after the ELM collapse. Sensitivity studies including the above case will be done and compared with experiments.

\section{Acknowledgements}

We are grateful to JT-60 and JET team members for collaboration. This work was partly supported by JSPS, Grant-in-Aid for Scientific Research. This work was also carried out under the collaborating research program at National Institute for Fusion Science. One of authors (N.H.) would like to thank Drs R. Ishizaki, Y. Tomita and A. Takayama for fruitful discussion and taking care of the NIFS program. N.H. is also grateful to Dr. M. Honda and Mr. I. Kamata for developing TOPICS-IB. This work was supported by EURATOM and carried out within the framework of the European Fusion Development Agreement. The views and opinions expressed herein do not necessarily reflect those of the European Commission.

\section{References}

[1] BAYLOR L.R. et al. 2007 Nucl. Fusion 471598

[2] KIM Ki Min et al. 2010 Nucl. Fusion 50055002

[3] LANG P.T. et al. 2008 Nucl. Fusion 48095007

[4] URANO H. et al. 2005 J. Plasma Fusion Res. 81280

[5] KOCSIS G. et al. 2007 Nucl. Fusion 471166

[6] KOCSIS G. et al. 2008 35th EPS Conf. on Plasma Physics (Hersonissos, Crete, Greece 2008) vol 32D (ECA) P-2.070

[7] SZEPESI T. et al. 2009 Plasma Phys. Control. Fusion 51125002

[8] WIESEN S. et al. 2008 Contrib. Plasma Phys. 48201

[9] HAYASHI N. et al. 2009 Nucl. Fusion 49095015

[10] KOECHL F. et al. 2010 37th EPS Conf. on Plasma Physics (Dublin, Ireland 2010) vol 34 (ECA) O4.123

[11] PARKS P.B., SESSIONS W.D. and BAYLOR L.R. 2000 Phys. Plasmas 71968

[12] SENICHENKOV I.Yu. et al. 2006 Nucl. Fusion 46788

[13] PEGOURIE B. et al. 2007 Nucl. Fusion 4744

[14] GAL K. et al. 2008 Plasma Phys. Control. Fusion 50055006

[15] ISHIZAKI R. et al. 2004 Phys. Plasmas 114064

[16] COMMAUX N. et al. 2010 Nucl. Fusion 50025011

[17] TAKENAGA H. and JT-60 Team 2001 Phys. Plasmas 82217

[18] LANG P. et al., ELM pacing investigations at JET with the new pellet launcher, this conference. 